\title{
Language and sociability: insights from Williams syndrome
}

\author{
Inna Fishman • Anna Yam • Ursula Bellugi • Debra Mills
}

Received: 30 November 2010 / Accepted: 29 May 2011 / Published online: 14 June 2011

(C) Springer Science+Business Media, LLC 2011

\begin{abstract}
One of the most compelling features of Williams syndrome (WS) is the widely reported excessive sociability, accompanied by a relative proficiency in expressive language, which stands in stark contrast with significant intellectual and nonverbal impairments. It has been proposed that the unique language skills observed in WS are implicated in the strong drive to interact and communicate with others, which has been widely documented in WS. Nevertheless, this proposition has yet to be empirically examined. The present study aimed at investigating the relationship between a brain index of language processing and judgments of approachability of faces, as a proxy for sociability, in individuals with WS as contrasted to typical controls. Results revealed a significant and substantial association between the two in the WS, but not in the control group, supporting the hitherto untested notion that language use in WS might be uniquely related to their excessive social drive.
\end{abstract}

Keywords Williams syndrome - Event-related potentials . N400 $\cdot$ Language $\cdot$ Sociability $\cdot$ Approachability

I. Fishman $(\bowtie) \cdot$ U. Bellugi

Laboratory for Cognitive Neuroscience,

The Salk Institute for Biological Studies, 10010 North Torrey Pines Road, La Jolla, CA 92037-1099, USA

e-mail: ifishman@mail.usf.edu

\section{A. Yam}

University of Florida,

Gainesville, FL, USA

D. Mills

Bangor University,

Bangor, UK

\section{Introduction}

Williams syndrome (WS) is a rare neurodevelopmental disorder caused by a hemizygous deletion of 25-30 genes on chromosome band 7q11.23 (Ewart et al. 1993) and associated with atypical social and cognitive profiles. Perhaps the most noted, albeit relatively little systematically investigated aspect of the WS behavioral profile is "sociability," a hallmark feature described as involving indiscriminate friendliness, enhanced empathy, and loquaciousness and socially engaging language (Doyle et al. 2004; Järvinen-Pasley et al. 2008; Jones et al. 2000; Mervis and Klein-Tasman 2000). The attraction to other people and interest in social interactions are evident even among infants and toddlers (cf. Järvinen-Pasley et al. 2008; Mervis et al. 2003) and persist into adulthood. Moreover, the noted gregariousness is often extended toward people who are judged by most other people as unapproachable (Bellugi et al. 1999; Järvinen-Pasley et al. 2010; Martens et al. 2009). Overall, notwithstanding the complex cognitive and behavioral profile of WS, excessive sociability and a keen interest in interacting with people are considered to be the most robust behavioral characteristics of WS (cf. Järvinen-Pasley et al. 2008).

In line with their prosocial behavioral orientation, individuals with WS show unusual language characteristics, which potentially enhance the likelihood of social communication with others. Namely, despite an overall level of intellectual functioning within the mild to moderate mental retardation range, individuals with WS appear to have relative proficiency in linguistic skills (cf. Mervis et al. 1999). Specifically, when compared with their mental-age and, often, chronological-age peers, WS individuals as a group are unusually loquacious and highly expressive (Udwin and Yule 1990) and give accurate and 
detailed verbal descriptions of objects (Bellugi et al. 1994) despite being unable to draw the same objects. On standardized vocabulary tests, WS subjects perform better than would be expected based on their mental age (see Brock 2007 for a review) and on fluency tasks they produce at least as many items as chronological-age controls, albeit generating more low-frequency words (Bellugi et al. 1994). There is also evidence to suggest that verbal abilities seem to develop at a faster rate in individuals with WS than their nonverbal abilities (Jarrold et al. 1998). Overall, notwithstanding the disagreements regarding the degree of impairment of the morphosyntactic aspects of language, which are beyond the scope of this article (see Karmiloff-Smith et al. 2003 for a review), it appears that linguistic skills are relative strengths in the WS cognitive profile, standing out in stark contrast to their overall intellectual disability and profound impairments in nonverbal, visuospatial cognitive functioning (Bellugi et al. 2000; Mervis et al. 1999).

Thus, together, there is a large corpus of literature indicating that verbal abilities and sociability, or attraction to other people, constitute key features of the cognitivebehavioral profile associated with WS. However, to date, no empirical evidence has been produced for the likely interplay between the WS language skills and their enhanced sociability. While attempts have been made to relate WS prosocial orientation to the other widely documented feature of their profile - an interest in attending to faces and overall strength in face processing (e.g., Mervis et al. 2003; Riby and Hancock 2008, 2009) - no explicit links have yet been made between their relatively proficient language and excessive social approach behavior. Hence, the aim of this study was to examine this relationship.

Pertinent to this goal, a recent study by Fishman et al. (2010) demonstrated that individuals with WS show evidence of enhanced brain activity associated with language processing, as measured by event-related potentials (ERPs). Specifically, in an auditory sentence task, the N400 ERP component, considered to be a robust marker of semantic processing, was found to be significantly larger in adults with WS than in typically developing (TD) controls and in individuals with autism (with overall cognitive abilities, as measured by IQ, ruled out as a potential confound). In the general population, the occurrence of a semantically incongruent word at the end of a sentence is associated with a large N400, a negativegoing potential occurring approximately $400 \mathrm{~ms}$ after the critical word onset (Kutas and Hillyard 1980). Overall, the N400 magnitude is thought to index the ease of integration of stimuli into an ongoing context (Kutas and Federmeier
2000) and serves as a metric of one's ability to process and extract meaning from linguistic stimuli (among other stimuli, see Kutas and Federmeier 2011) - i.e., a metric of language comprehension. Thus, the enhanced N400 effect found in WS by Fishman et al. suggests that individuals with WS allocate greater processing to extracting meaning from others' utterances, which could be due to a more elaborate or more densely connected semantic network - an interpretation consistent with behavioral evidence of surprisingly proficient performance on vocabulary and fluency tasks as reviewed above (but see Brock 2007 for a review of mixed behavioral findings on semantic fluency tasks in WS).

The present study was designed to build upon this finding in order to investigate the relationship between this electrophysiological correlate of language processing and enhanced sociability in WS. The N400 was chosen as an outcome measure of language processing due to its sensitivity to the processing of meaning (cf. Kutas and Federmeier 2011), which is a fundamental function of language. Overall, ERP measures - and the N400 in particular - are powerful tools for studying language and comprehension because they allow for tracking processing through time with millisecond resolution. Moreover, given the N400's functional specificity as a measure of brain language processing, it is especially well suited for studying language in special populations with limited abilities to meet complex task demands. Consequently, and in light of the evidence of the enhanced N400 effect in individuals with WS (Fishman et al. 2010), we chose to utilize the N400 ERP component as a measure of language processing to explore whether there exists an association between language and a widely reported excessive sociability WS. Due to the "special" status that both language and sociability occupy in the WS phenotype, we expected to find an association between these two domains in WS, but not in TD controls. Specifically, using the same ERP paradigm and stimuli as in Fishman et al., we predicted that, in individuals with WS, a larger N400 effect, as an electrophysiological index of language processing, would be positively correlated with the extent of their hypersociability. The sociability was assessed experimentally, with a standardized measure of ratings of approachability (Adolphs' approachability task) indexing one's willingness to approach unfamiliar people.

\section{Methods}

Participants

Sixteen individuals with WS (seven males; mean age, 22 years; range, 17-30 years) were recruited as part of 
an ongoing multicenter research program on WS. Genetic diagnoses of WS were established using fluorescent in situ hybridization test for elastin, a gene invariably associated with the WS microdeletion (Korenberg et al. 2000). Eighteen healthy individuals (eight males; mean age, 30 years; range, 19-41 years) were recruited through advertisements in local newspapers and fliers posted in the community and constituted the TD control group. All TD participants were screened for the level of education, and only those with no more than 2 years of college-level education were included in the study. All potential participants were screened for current and past psychiatric and/or neurological problems, including history of acquired brain injury and seizure disorder. All participants were native speakers of American English and had no hearing deficits. Demographic and psychometric information for each group is summarized in Table 1.

While a choice of an appropriate control group for WS is often controversial, this study was designed to explore how individuals with WS differ from those with typical development. Although the WS group was significantly younger than the TD group $(p<.001)$, a precise matching on chronological age was not our goal, given the WS neurocognitive deficits (furthermore, matching WS participants and controls on mental age, or IQ, would have resulted in a comparison group younger in age than the WS group, which would introduce a confound of age, such that potential differences on ERPs could be due to the age/maturation and not to the underlying cognitive processes). The purpose of recruiting an adult TD control group was to obtain a sample of typically developing, mature individuals to represent "typical" levels of sociability.

\section{Stimuli}

To elicit the N400, the well-tested anomalous sentence paradigm was employed. Participants listened to a series of 80 naturally spoken sentences, digitized at $12 \mathrm{kHz}$ and presented over headphones one word at a time, at a rate of one word/1,000 ms (for a complete description see Fishman et al. 2010). Half of the 80 sentences ended with a last word judged by an independent sample to be the best completion ending for that sentence (e.g., "Kids learn to read and write in school."), and half ended with an anomalous last word (e.g., "Kids learn to read and write in finger."), while retaining the same sentence stem. This set of sentences was originally devised so that each incongruent/anomalous sentence ending served as a congruent/best completion ending for another sentence (see Holcomb et al. 1992), thereby making the congruent and incongruent sets completely matching on all but semantic congruency attributes. The participant's task was to indicate, by a button press, whether or not the preceding sentence made sense.

\section{Procedure}

Language ERP task Participants were first exposed to ten practice trials, behavioral and electrophysiological responses to which were not included in later analyses. Each trial ended with an onset of a visual prompt, which followed $3 \mathrm{~s}$ after the final word and indicated that the participant should make a judgment. The prompt remained on the computer screen until the participant made a button response indicating whether or not the sentence made sense. This delayed response was designed to prevent any contamination of ERPs associated with processing of the final word by brain activity associated with the motor response. There was a 2- to 3-min self-paced break after every 20 trials.

Sociability measure: Adolphs' approachability task At the conclusion of the ERP testing, participants completed the modified version of the Adolphs' approachability task previously used with individuals with WS as a measure of sociability (Bellugi et al. 1999; Järvinen-Pasley et al. 2010; Martens et al. 2009). The task consisted of 42 blackand-white photographs of unfamiliar faces taken from an original set of 100 stimuli (Adolphs et al. 1998), 21 of which comprised the most positive ratings and 21 of

Table 1 Demographic and psychometric data for WS and TD groups

\begin{tabular}{|c|c|c|c|c|c|c|c|}
\hline Group & $N$ & $\begin{array}{l}\text { Gender } \\
(\mathrm{F} / \mathrm{M})\end{array}$ & $\begin{array}{l}\text { Age } \\
\text { (mean (SD; range)) }\end{array}$ & $\begin{array}{l}\text { Full scale } \\
(\text { mean }(\mathrm{SD}))\end{array}$ & $\begin{array}{l}\text { IQ: verbal } \\
(\text { mean }(\mathrm{SD}))\end{array}$ & $\begin{array}{l}\text { Performance } \\
\text { (mean (SD)) }\end{array}$ & $\begin{array}{l}\text { JLO } \\
(\text { mean }(\mathrm{SD}))\end{array}$ \\
\hline WS & 16 & $9 / 7$ & $22(3.9 ; 17-30)$ & $68(6.4)$ & $72(6.1)$ & $67(7.4)$ & $62(7.9)$ \\
\hline $\mathrm{TD}$ & 18 & $8 / 10$ & $30(7.5 ; 19-41)$ & $100(11.8)$ & $101(11.0)$ & $100(11.2)$ & 115 (15.0) \\
\hline
\end{tabular}

Note. The WS group was significantly younger and had lower IQ indices and visuospatial scores than the TD group (all $p$ values, $<.001$ ) 
which comprised the most negative ratings obtained from normal controls in the original Adolphs et al. study. The stimuli were presented individually in random order, while participants were asked to rate their approachability by considering "how much they would like to walk up and converse with the person in the photograph," using a 5point Likert scale ranging from $-2=$ "No" to $+2=$ "Yes," with $-1=$ "Probably not," $0=$ "Don't know," and $+1=$ "Maybe" (participants were presented with the color-coded verbal descriptors of the rating scale; the numerical values were not presented to simplify the scale utility for the WS participants). The number of Yes responses was tallied for each subject and served as a primary outcome measure. Mean ratings for 21 "positive" (i.e., trustworthy and approachable) and 21 "negative" (i.e., untrustworthy and unapproachable) faces, as rated by the original normative sample (Adolphs et al. 1998), were also calculated. Prior to administering the 42 test stimuli, participants were familiarized with the rating scale using a training set of faces.

Cognitive testing All participants were administered a battery of standardized cognitive tests, to determine whether the overall intellectual functioning and cognitive abilities have an effect on the predicted N400/sociability association. The Wechsler Adult Intelligence ScaleThird Edition (WAIS-III; Wechsler 1997), from which verbal, performance, and overall (i.e., full scale) intelligence quotients (VIQ, PIQ, and FSIQ) were derived, served as a measure of general cognitive ability and the Judgment of Line Orientation task (JLO) (Benton et al. 1983) served as a measure of visuospatial abilities. The IQ and JLO scores achieved by each group are displayed in Table 1. As was expected given the well-documented cognitive phenotype of WS, TD controls obtained significantly higher IQ and visuospatial scores than the WS group (all $t$ values (32)>9.32 and all $p$ values, <.01).

Electroencephalogram recording, off-line processing, and component extraction The electroencephalogram was recorded using a 16-electrode cap (ElectroCap International Inc., Eaton, Ohio) at $250 \mathrm{~Hz}$, with a bandpass of 0.01 to $100 \mathrm{~Hz}$. During data acquisition, all electrodes were referenced to A1 (right mastoid). The off-line processing steps, including component extraction strategy, were the same as those reported in Fishman et al. (2010). Briefly, after low-pass filtering at $30 \mathrm{~Hz}$ and re-referencing to an average mastoid reference, the data were subjected to an independent component analysis (Jung et al. 2000) for correction of ocular artifacts. Artifact-free data (a mean of $35 / 40$ trials, or $87.5 \%$, per condition) were segmented into 1,000-ms-long ERP epochs time-locked to the onset of the final word, baseline-corrected using a $100 \mathrm{~ms}$ baseline preceding the final word, and averaged separately for congruent and incongruent conditions. The N400 component was identified and quantified with a temporal principal components analysis (PCA), a factor-analytic statistical approach used with ERP data to produce objective, datadriven measurements of ERP components (cf. Spencer et al. 2001). ${ }^{1}$ The resulting PCA factor scores for the only factor loading highly in the $400 \mathrm{~ms}$ time window, as measured at vertex, or $\mathrm{Cz}$ electrode (in accordance with the wellestablished scalp distribution of the N400 elicited by auditory stimuli; cf. Kutas and Van Petten 1994), served as outcome variables. (No other factors yielded from the PCA were analyzed given the specificity of the a priori hypothesis and the task choice with regards to the N400.) The difference between the N400 factor scores for incongruent and congruent sentences (i.e., the N400 effect) served as a dependent variable.

\section{Results}

Analysis of the behavioral performance on the ERP task (i.e., accuracy of judgment whether the preceding sentence made sense or not) revealed that the WS group's mean accuracy $(96.1 \%, \mathrm{SD}=3.09)$ was not significantly different than that of TD $(96.7 \%, \mathrm{SD}=$ $5.69 ; t(32)=-.38$ and $p=.70)$. Despite the equivalent level of behavioral performance, the two groups exhibited significantly different N400 effects, as detailed

\footnotetext{
${ }^{1}$ The data matrix for the PCA consisted of voltage readings at each of the 250 time points $(1,000 \mathrm{~ms}$ epochs, sampled every $4 \mathrm{~ms})$ as variables and all the channels $(16) \times$ conditions $(2) \times$ participants (34) as cases. PCA decomposition was based on covariance association matrix. The number of components to be rotated was determined by the Scree test (Cattell 1966), which suggested retention of 15 factors accounting for $92.8 \%$ of the variance, which were then rotated to simple structure using the Varimax procedure (Donchin and Heffley 1978). The first temporal factor to emerge in PCA accounted for $44 \%$ of the variance and, given its shape and high loadings at the end of the epoch (see Fig. 2 in Fishman et al. 2010), appeared to reflect the classical slow wave, which typically emerges among the first factors in temporal PCAs (Spencer et al. 2001). The second factor accounted for $16 \%$ of the variance and loaded highly (factor loading of .90) in the $400 \mathrm{~ms}$ range, the time window where the differences between sentence categories and groups emerged in the averaged data, as seen in Fig. 1, left panel. Considering its latency range, this factor was deemed to represent the temporal activity associated with the N400. The third factor loaded highly in the $200 \mathrm{~ms}$ time window and accounted for $12 \%$ of the variance. The remaining factors had low (below .7) factor loadings and accounted for negligible amounts of variance.
} 
in Fishman et al. (2010), such that individuals with WS had significantly larger N400 effect than TD controls $(t$ $(32)=-2.74$ and $p=.01)^{2}$

The ratings for both positive and negative faces (as rated by the original Adolphs' normative sample) are presented in Table 2. Consistent with previous reports of the Adolphs' approachability ratings in WS (e.g., Bellugi et al. 1999; Järvinen-Pasley et al. 2010; Martens et al. 2009), a 2 (group) $\times 2$ (stimuli valence) mixed ANOVA with repeated measures of the valence (positive vs. negative) revealed that individuals with WS rated both positive and negative faces as more approachable than TD controls (main effect of valence, $F(1,32)=128.15$ and $p=0.001$ and group, $F(1$, $32)=13.8$ and $p=0.01$, but no significant valence by group interaction, $F(1,32)=1.29$ and $p>0.05) .{ }^{3}$ Reliability analysis revealed that both WS and TD groups showed high consistency of ratings of approachability across the stimulus items, with Cronbach's alpha of 0.81 for the WS and 0.93 for the TD group.

Correlation analyses (Pearson's $r$ tests) between the N400 and the approachability ratings, computed separately for each group, revealed significant correlations for the WS group $(r=-.54$ and $p=.02)$ but not for TD controls $(r=-.22$ and $p=.19)$. The negative correlation in WS signified that the larger the $\mathrm{N} 400$ (which is a negative component), the larger the proportion of Yes (i.e., I would approach this person) responses on the approachability task (see Fig. 1). A bootstrapped correlation analysis using 10,000 samples

\footnotetext{
$\overline{2}$ This difference did not reach significance when standard peak measures (peak amplitudes) were analyzed (WS mean amplitude $=-3.78(\mathrm{SD}=$ 3.64), TD mean amplitude $=-2.12(\mathrm{SD}=2.78)$, and $p=.054)$, further underscoring the need for uncontaminated, data-driven measurement of the ERP components such as PCA (since peak measures are subjects to such confounds as experimenter's bias and component overlap). Also, in keeping with the literature indicating general invariance of the N400 latency (as reviewed in Kutas and Federmeier 2011), no significant difference were found between the latency of the N400 peak between the two groups (as seen on the left panel of Fig. 1), with WS mean latency= $484.8(\mathrm{SD}=35.8)$ and TD mean latency=478.8 $(\mathrm{SD}=34.7), p=.64$.

${ }^{3}$ While two other studies of the approachability ratings in WS (Frigerio et al. 2006; Porter et al. 2007) produced different results, namely higher approachability ratings than the TD controls for positive face stimuli only (Frigerio et al. 2006), and similar approachability ratings to those of the TD controls (Porter et al. 2007), their data are not directly comparable to ours due to the significant differences in methodology. Specifically, the former study used (different) affective facial stimuli, displaying specific emotions such as anger, disgust, fear, happiness, etc., likely introducing a confound of affect, which is not trivial in this population given their well-documented perceptual deficits in affect identification; the latter study utilized additional input, such as voice and posture in addition to the facial stimuli, as well as repeated exposure to the stimuli-first to indicate the expression and then to rate the approachability of the face - both of which likely influenced perceptions and ratings of the approachability.
}

computed a $95 \%$ confidence interval ranging from -.17 to $-.79(\mathrm{SE}=0.12)$, indicating that this effect was robust and not driven by outlying values. Lack of a sizeable and significant correlation in the TD group, on the other hand, indicated that the association between the N400 and approachability was specific to WS.

Correlations were also calculated between the approachability ratings and other cognitive measures (IQ and JLO) to rule out a possibility that the positively skewed approachability ratings of the participants with WS may reflect impaired cognitive or visuospatial processing. There were no significant correlations (all $r$ values, $<.17$ and all $p$ values, $>.33$ ) between the approachability ratings and IQ or visuospatial scores, suggesting that intellectual or cognitive deficits did not account for the above effect. Likewise, and consistent with previous research (Fishman et al. 2010), there were no significant correlations between IQ (FSIQ, VIQ, or PIQ) and the N400 effect (all $r$ values, $<.10$ and all $p$ values, >.36). Finally, to rule out the potential confound of age, given the younger mean age of the WS group, the correlations between age and the two primary outcome variables (approachability ratings and N400) was examined. Pearson correlations assessed separately within each group revealed no significant results (all $r$ values, $<.17$ and all $p$ values, >.26).

\section{Discussion}

This study represents a first known-to-us attempt to examine the relationship between language and sociability in a syndrome characterized by unusual social interest and surprisingly proficient expressive language, the latter standing out in a cognitive profile characterized by overall intellectual disability and profound impairments in nonverbal, visuospatial functioning. The primary intent of the study was to assess the association between an electrophysiological correlate of language comprehension and approachability ratings, as a proxy for sociability, in individuals with WS as compared with TD controls, operating under the primary hypothesis that a relative proficiency in linguistic skills characterizing WS might be related to their strong drive to interact and communicate with others. In support of this hypothesis, the results revealed a significant and sizeable correlation between a brain index of language processing, as measured by the N400 component of the ERPs, and approachability ratings in individuals with WS, but not in TD controls. The larger the magnitude of the N400 effect, the higher was the positive bias in ratings of approachability of unfamiliar individuals in participants with WS, with bootstrap runs 
Table 2 Mean number of Yes responses and mean approachability ratings for positive and negative faces on Adolphs' approachability task for WS and TD groups

\begin{tabular}{|c|c|c|c|c|}
\hline & \multicolumn{2}{|c|}{ Total Yes responses ${ }^{\mathrm{a}}$} & \multicolumn{2}{|l|}{ Mean ratings ${ }^{b}$} \\
\hline & WS (mean (SD)) & TD (mean (SD)) & WS (mean (SD)) & $\mathrm{TD}($ mean $(\mathrm{SD}))$ \\
\hline Positive faces & $10.71(6.40)$ & $6.82(7.24)$ & $1.23(0.43)$ & $0.94(0.57)$ \\
\hline Negative faces & $1.82(3.60)$ & $1.42(1.41)$ & $-0.60(0.47)$ & $-0.70(0.75)$ \\
\hline
\end{tabular}

${ }^{\mathrm{a}}$ Total Yes responses=tally of all Yes responses given by each subject, separately for positive and negative faces

${ }^{\mathrm{b}}$ Ratings range from -2 (definitely would not want to talk to that person) to +2 (definitely would talk to that person)

showing that covariances between these variables were unlikely because of chance. This relationship, however, was absent in the control group. Further, consistent with previous studies utilizing the same approachability task (Bellugi et al. 1999; Martens et al. 2009), it was also found that individuals with WS gave higher approachability ratings to both "negative" and "positive" faces, as compared with controls.

Importantly, no association was found between age and approachability ratings or the N400 effect, for either group of participants. It was essential to rule out the potential confound of age, given that, first, the WS group was significantly younger than the TD group, and secondly, one might argue that with age, the extent of sociability as measured by approachability ratings may diminish in individuals with WS, as they internalize the frequent message from their family members to curb their indiscriminate approach behaviors (in the interest of their personal safety).

So, why would individuals with a syndrome characterized by relative strengths in expressive language report atypically positive ratings of the extent to which they would like to approach unfamiliar, and sometimes pre-judged as inapproachable strangers? At the level of cognitivebehavioral explanation, a key parameter of sociability is approaching new situations and people, which in humans often requires developed language skills. But this explanation is unsatisfactory by itself, because it does not account for the lack of such relationship in typically developing individuals. At the level of brain morphometry and anatomy, structural brain imaging evidence indicates that despite reduced total brain volume in WS, some areas have disproportionately large volumes, including the amygdala (Reiss et al. 2004). As is well documented elsewhere (cf. Adolphs 2001), the amygdala plays a critical role in social cognition, particularly in the perception of fear/danger, and in the subsequent regulation of appropriate behavioral and autonomic responses to social-affective stimuli-and thus, is likely involved in the approachability judgments. However, the N400 is known to arise from a highly distributed brain network (which includes superior/middle temporal gyrus, the temporo-parietal junction, and the medial temporal lobe, as well as some frontal regions; see Kutas and Federmeier 2011 for a review), making it imprudent to speculate about neuroanatomical bases for the approachability ratings/N400 association. ${ }^{4}$

From a theoretical perspective, this relationship suggests that the enhanced sociability as measured by higher ratings of approachability in individuals with WS extends to their use of language as indexed by the N400 component. This interpretation is in line with data demonstrating that individuals with WS frequently use a wide variety of affective and social engagement devices in their language, effectively using language for social purposes (Reilly et al. 2004). The social use of language in WS has been shown to extend across different cultures (the USA, France, and Italy; Reilly et al. 2005), demonstrating the robust nature of this feature in the WS profile. While the N400 does not measure this particular aspect of language per se, it does serve as a probe of the pathway sustaining language processing in the brain, including interpreting context and extracting meaning from what is said by others. Prior research (Fishman et al. 2010) has shown these processes indexed by the N400 to be uniquely enhanced in WS. The current finding provides some evidence that this enhanced language processing might be implicated in the bias toward seeking interactions with others observed in WS. Future studies should use other measures representing these two domains-language and hypersociability - to establish the generalizability of this finding.

Notably, these results may also be considered in light of the mirroring evidence emerging in individuals with

\footnotetext{
${ }^{4}$ Yet, it is noteworthy that cytoarchitectonic evidence points to relative preservation of cell density and cell size in the primary auditory cortex in WS (which, as was first suggested by Neville et al. 1994, may in part underlie the relative proficiency in language in WS), and an excessively large layer of neurons was found in an area receiving projections from the amygdala, suggesting that the auditory cortex may be more limbically connected in WS than in controls (Holinger et al. 2005).
} 
Fig. 1 Correlation between the approachability, Yes scores, and PCA-derived N400 effect, plotted separately by group. The ERP waveforms on the left illustrate the N400 effect (derived as an N400 factor score for incongruent sentences minus that for congruent sentences) in the WS and TD groups, which is plotted on the $y$-axis of the scatter plot on the right (the value of the N400 factor scores on the $y$-axis is a unitless dimension)
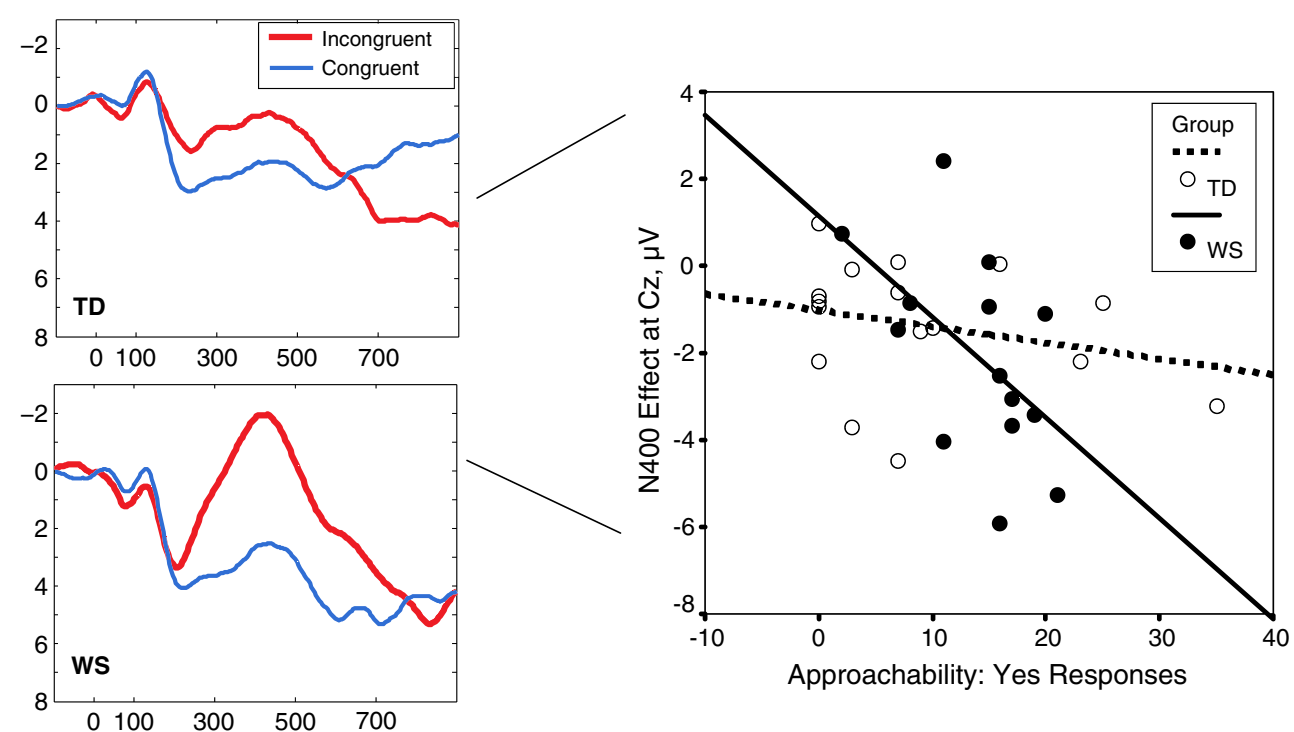

specific language impairment (SLI), who have been found to exhibit marked social difficulties (e.g., Hart et al. 2004). This condition provides another association -in the opposite direction - of inferior language abilities associated with diminished sociability (measured by Hart et al. via teacher ratings of sociable behaviors), suggesting that the two domains might be functionally linked. The mechanism through which language and sociability may be linked is a subject of an ongoing debate. Given that current theories postulate that, in SLI, these domains are linked via theory of mind mechanisms, future investigations in WS should employ measures compatible with theory of mind and tapping into language and sociability.

One possible limitation of the current study is a potential confound of cognitive disability in interpreting our results. Although the two main outcome measures - the N400 and the approachability ratings - were not correlated with IQ in our sample, the WS sample did not allow us to test this potential association in a full range of cognitive abilities, due to the fact that participants with WS fall on the lower end of the IQ continuum. Thus, the two groups together (WS and TD) did not cover a full range of IQ. Future work should utilize a broader range of control groups, including individuals with other developmental disorders, to overcome this potential limitation. In addition, in order to understand the developmental mechanisms giving rise to the language-hypersociability link in WS suggested by the current finding, longitudinal studies tracking developmental trajectories across these two domains, should be attempted next.

In sum, the present study contributes to the growing corpus of evidence demonstrating enhanced sociability in individuals with WS, utilizing an experimental approachability-judgment task. However, results of the present study extend these earlier findings by revealing a robust association between the "hypersociability" and atypical language skills as measured at the level of brain processing of linguistic information, both of which distinguish WS from other clinical and neurodevelopmental populations. This finding, in essence, supports the hitherto untested notion put forward by Reilly, Bellugi, and colleagues that unique expressive language skills characterizing individuals with WS are linked with their strong drive to interact and communicate with others (cf. Reilly et al. 2004, 2005). While we recognize the limited inference that can be made based on correlational results (for instance, with regards to causality), we also maintain the position that exploring individual variability, beyond summary statistics or analysis of variance at the group level, may be more beneficial in aiding our understanding of the core processes involved in WS social phenotype, with considerable implications for typical and atypical social function.

Acknowledgments We wish to thank all the participants and their families who so generously gave their time to take part in this study. This research was supported by a National Institute of Child Health and Human Development (NICHD) grant awarded to UB and DM and a National Institute of Mental Health (NIMH) fellowship awarded to IF. We thank Lawrence G. Appelbaum, Marie St. George, and Rowena Ng for their help with data collection and processing. We are especially grateful to Jyoti Mishra for her assistance with ERPSS programming.

\section{References}

Adolphs R. The neurobiology of social cognition. Curr Opin Neurobiol. 2001;11(2):231-9.

Adolphs R, Tranel D, Damasio AR. The human amygdala in social judgment. Nature. 1998;393:470-4.

Bellugi U, Wang P, Jernigan T. Williams syndrome: An unusual neuropsychological profile. In: Broman S, Grafman J, editors. Atypical cognitive deficits in developmental disorders: implications for brain function. Hillsdale: Erlbaum; 1994. p. 23-56. 
Bellugi U, Adolphs R, Cassady C, Chiles M. Towards the neural basis for hypersociability in a genetic syndrome. Neuroreport. 1999;8:1653-7.

Bellugi U, Lichtenberger L, Jones W, Lai Z, St. George M. I. The neurocognitive profile of Williams syndrome: a complex pattern of strengths and weaknesses. J Cogn Neurosci. 2000;12(Supplement 1):1-29.

Benton AL, Hamsher KdeS, Varney NR, Spreen O. Contributions to neuropsychological assessment: a clinical manual. New York: Oxford University Press; 1983.

Brock J. Language abilities in Williams syndrome: a critical review. Dev Psychopathol. 2007;19:97-127.

Cattell RB. The scree test for the number of factors. Multivar Behav Res. 1966;1(2):140-61

Donchin E, Heffley E. Multivariate analysis of event-related potential data: A tutorial review. In: Otto D, editor. Multidisciplinary perspectives in event-related potential research. Washington: US Government Printing Office; 1978. p. 555-72.

Doyle TF, Bellugi U, Korenberg JR, Graham J. "Everybody in the world is my friend": hypersociability in young children with Williams syndrome. Am J Med Genet. 2004;124A:263-73.

Ewart AK, Morris CA, Atkinson D, Jin W, Sternes K, Spallone P, et al. Hemizygosity at the elastin locus in a developmental disorder, Williams syndrome. Nat Genet. 1993;5(1):11-6.

Fishman, I., Yam, A., Bellugi, U., Lincoln, A., \& Mills, D. (2010). Contrasting patterns of language-associated brain activity in autism and Williams syndrome. Social Cognitive and Affective Neuroscience, doi:10.1093/scan/nsq075.

Frigerio E, Burt DM, Gagliardi C, Cioffi G, Martelli S, Perrett DI, et al. Is everybody always my friend? Perception of approachability in Williams syndrome. Neuropsychologia. 2006;44:254 59.

Hart K, Fujiki M, Brinton B, Hart CH. The relationship between social behavior and severity of language impairment. J Speech Lang Hear Res. 2004;47:647-62.

Holcomb P, Coffey S, Neville H. Visual and auditory sentence processing: a developmental analysis using event-related brain potentials. Dev Neuropsychol. 1992;8(23):203-41.

Holinger DP, Bellugi U, Mills DL, Korenberg JR, Reiss AL, Sherman GF, et al. Relative sparing of primary auditory cortex in Williams syndrome. Brain Res. 2005;1037:35-42.

Jarrold C, Baddeley AD, Hewes AK. Verbal and nonverbal abilities in the Williams syndrome phenotype: evidence for diverging developmental trajectories. J Child Psychol Psychiatry. 1998;39:511-23.

Järvinen-Pasley A, Bellugi U, Reilly J, Mills DL, Galaburda A, Reiss AL, et al. Defining the social phenotype in Williams syndrome: a model for linking gene, brain, and cognition. Dev Psychopathol. 2008;20:1-35.

Järvinen-Pasley A, Adolphs R, Yam A, Hill KJ, Grichanik M, Reilly J, et al. Affiliative behavior in Williams syndrome: social perception and real-life social behavior. Neuropsychologia. 2010;48:2110-9.

Jones W, Bellugi U, Lai Z, Chiles M, Reilly J, Lincoln A, et al. II. Hypersociability in Williams syndrome. J Cognit Neurosci. 2000;12(Supplement 1):30-46.

Jung TP, Makeig S, Westerfield M, Townsend J, Courchesne E, Sejnowski T. Removal of eye activity artifacts from visual eventrelated potentials in normal and clinical subjects. Clin Neurophysiol. 2000;111(10):1745-58.

Karmiloff-Smith A, Brown JH, Grice S, Paterson SJ. Dethroning the myth: cognitive dissociations and innate modularity in Williams syndrome. Dev Neuropsychol. 2003;23:227-43.
Korenberg JR, Chen X-N, Hirota H, Lai Z, Bellugi U, Burian D, et al. VI. Genome structure and cognitive map of Williams syndrome. J Cognit Neurosci. 2000;12(Supplement 1):89-107.

Kutas M, Federmeier KD. Electrophysiology reveals semantic memory use in language comprehension. Trends Cogn Sci. 2000;4:463-70.

Kutas M, Federmeier KD. Thirty years and counting: Finding meaning in the N400 component of the event-related brain potential (ERP). Annu Rev Psychol. 2011;62(14):1-27.

Kutas M, Hillyard SA. Reading senseless sentences: Brain potentials reflec semantic incongruity. Science. 1980;207:203-5.

Kutas M, Van Petten C. Psycholinguistics electrified: Event-related brain potential investigations. In: Gernsbacher MA, editor. Handbook of psycholinguistics. San Diego: Academic; 1994. p. 83-143.

Martens MA, Wilson SJ, Dudgeon P, Reutens DC. Approachability and the amygdala: insights from Williams syndrome. Neuropsychologia. 2009;47:2446-53.

Mervis CB, Klein-Tasman BP. Williams syndrome: cognition, personality, and adaptive behavior. Ment Retard Dev Disabil Res Rev. 2000;6:148-58.

Mervis CB, Morris CA, Bertrand J, Robinson BF. Williams syndrome: findings from an integrated program of research. In: TagerFlusberg H, editor. Neurodevelopmental disorders. Cambridge, MA: MIT Press; 1999. p. 65-110.

Mervis CB, Morris CA, Klein-Tasman BP, Bertrand J, Kwitny S, Appelbaum LG, et al. Attentional characteristics of infants and toddlers with Williams syndrome during triadic interactions. Dev Neuropsychol. 2003;23:243-68.

Neville HJ, Mills DL, Bellugi U. Effects of altered auditory sensitivity and age of language acquisition on the development of languagerelevant neural systems: preliminary studies of Williams Syndrome. In: Broman SH, Grafman J, editors. Atypical cognitive deficits in developmental disorders. Hillsdale: Erlbaum; 1994. p. 68-83.

Porter MA, Coltheart M, Langdon R. The neuropsychological basis of hypersociability in Williams and Down syndrome. Neuropsychologia. 2007;45:2839-49.

Reilly J, Losh M, Bellugi U, Wulfeck B. "Frog, where are you?" Narratives in children with specific language impairment, early focal brain injury and Williams syndrome. Brain Lang. 2004;88:229-47.

Reilly J, Bernicot J, Vicari S, Lacroix A, Bellugi U. Narratives in children with Williams syndrome: a cross linguistic perspective. In: Ravid D, Bat-Zeev Shyldkrot H, editors. Perspectives on language and language development: essays in honor of Ruth A. Berman. Dordrecht: Kluwer; 2005. p. 303-12.

Reiss AL, Eckert MA, Rose FE, Karchemskiy A, Kesler S, Chang M, et al. An experiment of nature: brain anatomy parallels cognition and behavior in Williams syndrome. J Neurosci. 2004;24:5009-15.

Riby DM, Hancock PJ. Viewing it differently: social scene perception in Williams syndrome and autism. Neuropsychologia. 2008;46:2855-60.

Riby DM, Hancock PJ. Looking at movies and cartoons: eye-tracking evidence from Williams syndrome and autism. J Intellect Disabil Res. 2009;53:169-81.

Spencer KM, Dien J, Donchin E. Spatiotemporal analysis of the late ERP responses to deviant stimuli. Psychophysiology. 2001;38:343-58.

Udwin O, Yule W. Expressive language of children with Williams syndrome. Am J Med Genet. 1990;6(Suppl):108-14.

Wechsler D. Wechsler Adult Intelligence Scale - third edition (WAISIII). San Antonio: Psychological Corporation; 1997. 